\title{
El paradigma neoliberal de gobierno: precarización, inmunidad y control
}

\section{POR CANDELA DE LA VEGA $\left({ }^{*}\right)$ Y MARÍA ALEJANDRA CIUFFOLINI $\left({ }^{* *}\right)$}

Sumario: I. Introducción.- II. Estado de inseguridad: gestión de desigualdades, riesgo y creatividad/consumo.- III. El estado vigilante.IV. Reflexiones finales: inmunidad, política y derecho.- V. Bibliografía.

Resumen: situadas desde la preocupación por la gubernamentalidad neoliberal, este artículo parte de la pregunta sobre cómo es que se gobierna, a través de qué procesos y relaciones el gobierno se vuelve posible. A partir de resultados de estudios previos, pretendemos articular una respuesta a tal pregunta, desde las coordenadas actuales de nuestras desiguales sociedades latinoamericanas. Así, sostenemos que las formas de gobierno actuales dan origen a lo que ya Lorey llamó un Estado de Inseguridad, que se basa en un campo de apropiación individual de beneficios y de socialización de daños para crear un escenario de producción y gestión de la precariedad. Pero como la policéfala Hidra de Lerna de la mitología griega, ese Estado de Inseguridad tiene como segunda cabeza un Estado Vigilante, que despliega una cada vez más densa vigilancia del espacio público y cada vez

$\left(^{*}\right)$ Doctora en Ciencias Sociales, Universidad de Buenos Aires (UBA). Magíster en Administración Pública, Universidad Nacional de Córdoba (UNC) y Lic. en Ciencia Política, Universidad Católica de Córdoba (UCC). Investigadora de la Universidad Nacional de Córdoba y de la Universidad Católica de Córdoba. Becaria Posdoctoral del CONICET. Prof. de Teoría Política Contemporánea y de Análisis de Políticas Públicas, Universidad Católica de Córdoba. Desde 2004 integra el Equipo de Investigación "El llano en llamas".

(**) Posdoctora, Universidad Nacional de Córdoba (UNC). Doctora en Ciencias Sociales, Universidad de Buenos Aires (UBA). Magíster en Administración Pública, Universidad Nacional de Córdoba (UNC) y Lic. en Relaciones Internacionales, Universidad Católica de Córdoba (UCC). Investigadora de la Universidad Nacional de Córdoba y de la Universidad Católica de Córdoba. Prof. de Teoría Política Contemporánea y Epistemología de las Ciencias Sociales, Universidad Católica de Córdoba y de Sociología Jurídica, Universidad Nacional de Córdoba. Prof. de diversos programas de posgrado. 
más preciso el control sobre los movimientos de los individuos, lo que delimita nuevas coordenadas para lo común o lo colectivo.

Palabras claves: neoliberalismo - gubernamentalidad - precarización - vigilancia

\section{The neoliberal paradigm of government: precariousness, immunity and control}

Abstract: located from the preoccupation about neoliberal governmentality, this article starts from the question of how we are governed, through which processes and relationships government becomes possible. Based on the results of previous studies, we intend to articulate an answer to this question, from the current coordinates of our unequal Latin American societies. Thus, we maintain that the current forms of government give rise to what Lorey already called a State of Insecurity, which is based on a field of individual appropriation of benefits and socialization of damages to create a scenario of production and management of precariousness. But like the polycephalous Hydra of Lerna in Greek mythology, that State of Insecurity has as its second head a Vigilant State, which deploys an increasingly dense surveillance of public space and more and more precise control over the movements of individuals, what that delimits new coordinates for the common or the collective.

Keywords: neoliberalism - governmentality - precarization - surveillance

\section{Introducción}

El paradigma moderno de la política erigió al tema de la soberanía, el poder y el derecho en el núcleo del hacer político, y en ellos enraizó las posibilidades de la emancipación. En ese marco, la gubernamentalidad - el arte de gobernar, es decir, el conjunto de prácticas tendientes a poner límites al campo de acciones posibles para hombres y mujeres- se desplegaba intrínsecamente ligada a dispositivos de representación -ideológicos, principalmente- que diagramaban la modalidad de ejercicio del poder, de la relación entre gobernados y gobernantes, de constitución de las relaciones políticas y de la forma Estado.

Ese orden de representación es el que la racionalidad neoliberal ha disuelto a través de la suplantación de la política y sus categorías clásicas, por ejemplo, las de ciudadanía, derechos o soberanía. En su lugar, la racionalidad neoliberal proyecta el espacio configurativo del orden político desde la utilidad, esto es, la maximización del interés en las distintas esferas de la vida social (Brown, 2015; Ciuffolini, 2017a; De Lagasnerie, 2015; Laval y Dardot, 2013).

Dicha transfiguración trae consigo, por un lado, un aspecto muy estudiado actualmente que es la nueva configuración subjetiva. Asumiendo que gran parte de 
la fuerza del capitalismo reside en su capacidad de articular una organización de la producción de bienes y servicios con la producción de subjetividades, distintos análisis ubican el reemplazo del sujeto de derecho en sujeto de interés (1) como supuesto de las estrategias de gobierno neoliberales. Pero, por otro lado, en una perspectiva más amplia, la gubernamentalidad neoliberal exige abrir el campo para descubrir una modulación de las relaciones políticas entre gobernados y gobernantes y de la forma Estado. Es que, siguiendo a Foucault (2006), el Estado siempre es una resultante de la forma de gobernar que caracteriza, históricamente, a una sociedad y a una época. De ahí la importancia metodológica por responder, en primer lugar, a la pregunta sobre cómo es que se gobierna, a través de qué procesos y relaciones el gobierno se vuelve posible.

Este artículo pretende articular una respuesta a tal pregunta desde las coordenadas específicas de nuestras sociedades latinoamericanas actuales. La principal tesis que sostenemos es que, articulados a partir de un poder concebido como norma oblicua destinada a reconducir escenarios estratégicos, las formas de gobierno actuales dan origen a lo que ya Lorey (2016) llamó un Estado de Inseguridad, que se basa en un campo de apropiación individual de beneficios y de socialización de daños para crear un escenario de producción y gestión de la precariedad. Pero como la policéfala Hidra de Lerna de la mitología griega, ese Estado de Inseguridad tiene como segunda cabeza un Estado Vigilante, que despliega una cada vez más densa vigilancia del espacio público y cada vez más preciso el control sobre los movimientos de los individuos, lo que delimita nuevas coordenadas para lo común o lo colectivo.

Metodológicamente, estas afirmaciones teóricas se sustentan en los resultados de más de 15 años de investigación del equipo de investigación "El Llano en llamas" (2). Especialmente en los últimos años, nuestros trabajos previos vienen mostrando que la expansión del orden neoliberal nos situó frente a un nuevo repertorio de conceptos y formas de percepción en relación al mercado; a las formas

(1) En general, estos estudios se basan en la afirmación de Foucault (2007) de que el sujeto de interés "desborda al sujeto de derecho" (p. 315), "el primero acepta la limitación, la obediencia a la ley; mientras que el segundo no renuncia jamás a su interés, no detiene el proceso de maximización de su utilidad en nombre de exigencias presentadas como superiores" (p. 334-335).

(2) El equipo de investigación "El llano en llamas" es un equipo que trabaja en la Universidad Católica de Córdoba (UCC) y en la Universidad Nacional de Córdoba (UNC) desde el año 2004 con análisis sobre conflictos y luchas sociales contemporáneas. Desde el año 2019, la Secretaría de Investigación de la Universidad Católica de Córdoba (UCC) acreditó y financió el proyecto denominado "La racionalidad neoliberal, el gobierno de la vida y la reconfiguración de lo común como espacio de resistencia en Argentina, 2002-2017" (Res. No 1363/19). A su vez, desde el año 2018 la Secretaria de Ciencia y Técnica (SECyT) de la UNC también aprobó el proyecto denominado "Precarización, gobierno de la vida y resistencias. Un estudio de los conflictos políticos y sociales en Córdoba" (Res № 411/2018). 
de intervención del Estado y su sentido de legitimidad; a la propiedad de uno mismo, de nuestro cuerpo y de los cuerpos otros; de los territorios y de la naturaleza, de las maneras de vivir, producir y re-producirnos; y, finalmente, de las formas de subjetividad política (3). Integrando y retomando estos resultados es que pudimos encontrar ciertas regularidades en la estrategia gubernamental y es en ese sentido que podemos hablar de una racionalidad neoliberal de gobierno que se despliega en nuestras sociedades latinoamericanas del siglo XXI, atravesadas por una histórica pero renovada matriz capitalista y un modelo de desarrollo que actualiza las dinámicas del colonialismo extractivo y predatorio.

Este artículo se organiza en dos apartados principales. En el primero desarrollamos la primera de estas cabezas, el Estado de Inseguridad. En el segundo apartado, el Estado Vigilante. Finalmente, ofrecemos algunas reflexiones finales de cara a desandar caminos de resistencia, solidaridad, democracia e igualdad.

\section{Estado de Inseguridad: gestión de desigualdades, riesgo y creatividad/ consumo}

Coincidimos con algunos autores (Fraser, 2017; Lazzarato, 2015 y 2013; Lorey, 2016; de Lagasnerie, 2015) en que, alrededor del mundo, y desde la década de 1970, el capitalismo comenzó a organizarse en una escala global a partir de una lógica neoliberal que, basada en la generalización del mercado, la utilidad y la competencia, reestructuró transversalmente el gobierno de las relaciones sociales. El orden neoliberal no es una alternativa al capitalismo, es una manera de "hacerlo funcionar" (Foucault, 2007, p. 111). Laval y Dardot (2013) afirman que, justamente, el neoliberalismo es tanto más resiliente mientras más excede la esfera mercantil y financiera donde reina el capital, produciendo así una suerte de parámetro de interpretación para una multiplicidad de situaciones de vida. Más aún, explica de Lagasnerie (2015), por esta disposición transversal, la lógica neoliberal actúa hoy como factor de erradicación de los clásicos clivajes teóricos y políticos — socialismo/capitalismo; derecha/izquierda; por caso-. En lugar de "desencadenar una multiplicidad de interpretaciones contradictorias, genera sentimientos análogos en personas (o discursos) de las que habría cabido esperar la adopción de posiciones alejadas y hasta opuestas" (de Lagasnerie, 2015, p. 14)(4).

(3) Remitimos, especialmente, a los siguientes resultados de estudios previos: de la Vega et. al. (2020), de la Vega (2020; 2019; 2017; 2010); Ciuffolini (2017a y 2017b); Ciuffolini y de la Vega (2019); Avalle (2017); Azarian, Villegas y Whitney (2017); Azarian (2016); Saccucci (2018). Otros resultados pueden consultarse en www.llanocordoba.com.ar

(4) Laval y Dardot (2013) agregan que el neoliberalismo es mucho más que una ideología partidaria: el neoliberalismo, cuando inspira políticas concretas, se niega a sí mismo como ideología porque es la razón misma. En consecuencia, políticas muy parecidas pueden recibir moldes distintos (con- 
Por esta capacidad de instituirse como forma de vida - sin que importe demasiado la forma del régimen político-, América Latina es el espacio social donde el neoliberalismo adquirió las notas más radicales si consideramos los impactos en la reorganización del Estado, el mercado, la política y la sociedad. El escenario latinoamericano del siglo XXI muestra con claridad el efecto devastador de varias décadas de aplicación y vigencia de la matriz capitalista y neoliberal: disminución del PBI(5), concentración de riqueza, aumento de la deuda externa, inestabilidad económica e institucional y la consolidación de un modelo de desarrollo que actualiza las dinámicas del colonialismo extractivo y predatorio (Acosta, 2016; Azamar Alonso y Carrillo González, 2017; Composto y Navarro, 2014; Gago y Mezzadra, 2015). Particular mención merece la intensificación del patrón de desigualdad social, cuyas dimensiones determinantes exceden los ingresos y el nivel socioeconómico, y, como sugiere CEPAL incluye:

a) las desigualdades de género; b) las desigualdades étnicas y raciales; c) las desigualdades relacionadas con el ciclo de vida (especialmente en la infancia, la juventud y la vejez), y d) las desigualdades territoriales (entre las zonas rurales y urbanas y entre las distintas regiones de cada país). Se señala también que esas diversas dimensiones con frecuencia se encadenan, se entrecruzan y se potencian entre sí, afectando en forma particular a determinados grupos de población, como las mujeres indígenas y las mujeres afrodescendientes (CEPAL, 2016, p. 11).

En este escenario general, la tesis que sostenemos en este apartado es que la racionalidad neoliberal se desenvuelve patentemente como gestión diferencial de las desigualdades en un marco general más general de gobierno que instituye la inseguridad como preocupación principal de los sujetos.

Comenzar a explicar lo anterior supone partir de considerar una serie de desplazamientos y rupturas con el paradigma bienestarista del Estado Social. Tal como ya advertimos en un artículo previo (Ciuffolini, 2017a), este paso de una racionalidad a otra no es algo inmediato, ni lineal. Al contrario, es un proceso con marchas y contra marchas, en las que ambas se solapan y se tensionan. Incluso los efectos específicos de la racionalidad neoliberal se modulan en ese espacio abierto por las tensiones y las disputas del presente.

servadores, tradicionalistas, modernistas, republicanas, en función de las situaciones y los casos), poniéndose así de manifiesto su extrema plasticidad.

(5) Según la CEPAL (2020), la región viene registrando una trayectoria de bajo crecimiento desde fines de la década de 1970. Una comparación histórica por década muestra que el período 2010-2019 es el de menor crecimiento de la región en siete décadas: entre 2010 y 2019 la tasa de crecimiento del PIB regional disminuyó del $6 \%$ al 0,1\%. 
¿De qué desplazamientos estamos hablando? En primer lugar, de la suspensión de la igualdad como principio regulador y compensador de los desequilibrios e inequidades del mercado. Como modo de gobierno de una específica configuración capitalista, las instituciones protectoras del Estado de Bienestar, apoyadas en el principio de la igualdad, exigían formas de redistribución de recursos - económicos y políticos - tuteladas por el Estado y, a menudo, positivamente especificados bajo la forma de derechos. El progresivo desmantelamiento de tales instituciones significó, tal como sugiere Dávilo (2018), la disfuncionalidad de esa forma de gobernar en relación a las formas de acumulación capitalista global. Decíamos en un artículo previo (Ciuffolini, 2017a) que si la política de bienestar puede resumirse en el diseño de contrapesos o compensaciones a procesos económicos salvajes respecto a los cuales se admite que, en sí mismos, van a inducir efectos de desigualdad y provocar perjuicios sociales que pueden resultar destructivos y desestabilizadores; ahora, se trata de introducir el principio de mercado como regulación de la sociedad, unificando así los más diversos regímenes de existencia. Ello implica que la lógica de la competencia se proyecte ya como dimensión epistemológica - es decir, como umbral de inteligibilidad para las acciones individuales y colectivas-, ya como una dimensión ética —es decir, un estándar de evaluación de conductas- (Foucault, 2007).

Que la estrategia gubernamental se siente sobre un individuo que compite a través de la inversión en sí mismo no implica que se abandone "lo social" como ámbito de ejercicio del gobierno. Pero ya no será la desigualdad sino la pobreza lo que permite recortar el segmento social al que atenderá el Estado (Dávilo, 2018; Laval y Dardot, 2013). En el marco neoliberal, la pobreza es considerada el resultado de las "dificultades" que enfrentan algunos grupos para acumular, en sí mismos, diversas formas de capital (por ejemplo, la dificultad de no invertir de manera suficiente en el propio capital humano a través de la educación). Es que el neoliberalismo rechaza la idea de la protección indiscriminada de todos los ciudadanos y ciudadanas y exalta el valor de la "lucha personal". Si el éxito o el fracaso personal son interpretados como virtudes empresariales o fallas personales, se comprende que, más que esfuerzos de redistribución, se trata de "capacitar emprendedores"; más que solidaridad y compromiso cívico, se trata de "ayudar" a poblaciones específicas.

El segundo desplazamiento nos sitúa frente al primado de la libertad —entendida en la fórmula neoliberal de ausencia de coacción (Gray, 1994; Hayek, 2008)— como único principio regulador del orden social y la reproducción capitalista. Ello se corresponde con la configuración de un individuo "imbatible" o "resiliente" (6),

(6) El término fue acuñado por Emmy Werner y Ruth Smith en su libro "Vulnerable but Invencible. A Longitudinal Study of Resilient Children and Youth", en 1955. 
cuya cualidad es la elasticidad, la plasticidad, la autorregulación; o, en otras palabras, su capacidad de enfrentar y adaptarse a cualquier tipo de circunstancias adversas. Un individuo capaz de imaginar un mundo feliz a pesar del sufrimiento, pareciera ser el imperativo de la época (7). Pero, librados cada vez más a sus propios recursos y a su propia sagacidad, los individuos se ven obligados a idear gestionar soluciones individuales frente a problemas generados socialmente. Como explica Lazzarato (2013), el trabajo y el trabajo sobre sí mismo, reduce este último a una exhortación a ser, uno o una, su propio patrón o patrona, en el sentido de "hacerse cargo" de los costos y los riesgos que la empresa y el Estado externalizan en la sociedad.

\section{Explica el mismo autor que}

La promesa de lo que 'trabajo sobre sí mismo' debía aportar al 'trabajo' en términos de emancipación (goce, realización, reconocimiento, experimentación de formas de vida, movilidad, etc.) se ha invertido, para transformarse en el imperativo de hacer propios los riesgos y los costos que ni las empresas ni el Estado quieran asumir (Lazzarato, 2013, p. 108).

La paradoja de este individuo imbatible es que se configura en un contexto en el que la inseguridad es una condición existencial generalizada. Los cambios que producen las regulaciones a favor del libre desarrollo del interés y la utilidad tienen como correlato la intensificación de la fabricación de riesgos universalizados, ya se trate del cambio climático, de los virus biológicos y/o informáticos, del contagio bursátil o del terrorismo. Esta dinámica de apropiación individual de beneficios y socialización de daños no es algo novedoso: ha sido la mecánica histórica del capitalismo. La apropiación individual de beneficios y socialización de daños es el sustrato sobre el que se asienta de manera renovada la acumulación capitalista, mas su particularidad en el presente es el alcance de la destrucción y su inmediatez.

Para entender lo anterior es necesario atender el hecho de que, bajo el orden neoliberal, las energías de los individuos están dispuestas en dos sentidos. Primero, en la creatividad que requiere el despliegue e invención de estrategias de vida para sobrevivir, o para resistir; segundo, la satisfacción inmediata frente a la incertidumbre, que se expresa en el crecimiento exponencial de las dinámicas de consumo, convertido en clave para la movilización de un capitalismo impulsado por

(7) No obstante, como dice Torres (2019) la felicidad se promueva en casi todos los escenarios sociales, aun cuando el sujeto actual vive en la más profunda crisis experiencial y existencial: la felicidad parece una nueva promesa mesiánica en cuya búsqueda, el sujeto contemporáneo parece haberse perdido. 
la demanda y las necesidades del consumidor (Lazzarato, 2015; Harvey, 2020) (8). En esta dinámica ineludible que exige de los individuos "resistencia-creatividadconsumo" se anclan los más novedosos procesos de extracción de valor para el capital y, al mismo tiempo, el sustrato que promueve la singularidad individual y la diferenciación social a partir de un arte de vivir y sobrevivir. A su vez, la exaltación de la diferencia y de la singularidad propicia un nuevo modo de clasificación que da cuenta de un patrón inestable y móvil, en el que grupos e individuos se ordenan como incluidos o excluidos. Pero devenir excluido no es ya una condición para grupos determinados, sino una condición potencial para cualquiera. La posibilidad de la exclusión es entonces un problema para los otrora "protegidos" por el Estado en el capitalismo bienestarista. La novedad es que la precariedad es ahora un devenir plausible para los sectores medios - pues los pobres han revestido esa condición desde siempre- y para los individuos sin distinción.

En palabras de Lorey (2016)

Ser considerado como alguien que pertenece a un interior o a un exterior, o considerarse tal, no es para Castel una disyuntiva, sino más bien un camino procesual entre zonas. Antes que un límite estricto, imagina una especie de umbral de ambivalencia entre inclusión y exclusión, entre la 'zona de integración' y la de 'desafiliación.' La 'zona intermedia, inestable' es la de la 'precariedad', la inseguridad y la fuente de peligro. La 'precariedad' corresponde a una nueva forma de inseguridad que cabe atribuir al desmoronamiento y la disolución de las estructuras protectoras que se habían desarrollado dentro de la sociedad del trabajo asalariado. Por consiguiente, cabe hablar (...) de una inseguridad que continúa viéndose rodeada y penetrada por estructuras de protección (pp. 64-65).

Lazzarato (2013), Harvey (2020) y Hardt y Negri (2020) coinciden que esta precarización es el nuevo nombre de una antigua realidad: la proletarización a gran escala. La resistencia para sobrevivir, la creatividad y la singularidad son el lugar de anclaje de la extracción de valor en la gestión diferencial de las desigualdades bajo la racionalidad neoliberal. Pero la precarización generalizada también forma parte de los mecanismos a partir de los cuales se despliega la violencia y/o se normaliza a los individuos. Es que, cuando la inseguridad es la norma para los

(8) Harvey (2020) afirma que entre el 70 y el 80\% de las economías capitalistas están movidas por el consumo y advierte cómo se han estrellado, con la actual crisis epidemiológica por COVID 19, los modos de consumismo que explotaron después de 2007-8, especialmente para sectores como el turismo, hotelería y transporte aéreo. El consumo, según Lazzarato (2013), forma parte de unos de los principales mecanismos en los que se asienta la economía de la deuda, como motor económico y subjetivo de la economía contemporánea. 
sujetos, se prepara el terreno para la necesidad de regulación y de inmunización ante el riesgo.

\section{El Estado vigilante}

Pero la gestión neoliberal de los individuos y las poblaciones combina precariedad con un paradigma inmunitario del ejercicio del gobierno que habilita el despliegue de estrategias de control. La imbricación entre política y biología o derecho y biología, es un proceso que ya lleva más de tres siglos. Tal como dice Foucault (2008), el mismo ha constituido el cimiento de la arquitectura políticojurídica moderna y sus modulaciones a lo largo del tiempo han dado lugar tanto a regímenes totalitarios y trágicos, como al despliegue de un orden jurídico democrático, que nos resguarda y limita la arbitrariedad del poder. En uno y otro sistema - aunque de manera diferenciada - anida la idea de inmunidad como paradigma de la relación entre individuo y comunidad (Espósito, 2011).

En los regímenes totalitarios, inmune no remite necesariamente a una polaridad semánticamente contraria al léxico de la comunidad, como afirma Espósito (9). En ellos la constitución de lo común (raza, religión, ideología, nación, etc.) es la condición a partir de la cual se inmuniza una parte del cuerpo social frente a "otros" peligrosos, que pueden ser desafiliados o exterminados arbitrariamente. En la métrica de la racionalidad neoliberal, el movimiento es inverso: el individuo se inmuniza de lo común y lo colectivo. Esa tendencia hacia la atomización desconoce la condición de extrema interdependencia entre los seres vivientes, y por lo tanto sus efectos en la comunidad son catastróficos(10). Sin embargo, en ambos regímenes, encontramos un devenir similar de los paradigmas inmunitarios: la limitación de las libertades impuesta por los gobiernos se acepta en nombre de un deseo de seguridad que ha sido inducido por los mismos gobernantes, que intervienen activamente para satisfacerlo. Así, la excepción se convierte en regla y las instancias de coordinación o cooperación se activan frente a la amenaza, se trate de un desastre natural, un virus informático o biológico de alcance global, el contagio en las bolsas a nivel internacional, el terrorismo, etc. La amenaza es la

(9) Según Espósito (2020), el término "viral” indica una contaminación biopolítica entre diferentes lenguajes — políticos, sociales, médicos, tecnológicos- unificados por el mismo síndrome inmune, entendido como una polaridad semánticamente contraria al léxico de la comunidad.

(10) En este sentido, los desarrollos de Foucault (2007) sobre la biopolítica como estrategia gubernamental del liberalismo nos advierten que el concepto de "población" — objeto de la biopolítica- define un colectivo compatible con la ida de liberal de sociedad integrada por individuos calculadores y maximizadores de beneficio, en la medida en que lo que articula a los individuos son las características inherentes a su condición biológica y sus ciclos vitales — nacimiento, enfermedad, reproducción, muerte-, mas no el compromiso cívico con una comunidad de pertenencia o el vínculo identitario o nacional. 
condición o circunstancia que los gobiernos esgrimen para activar mecanismos de cooperación y/o coordinación, en el contexto de una racionalidad neoliberal que trabaja desde las premisas del individualismo y el orden espontáneo.

La crisis epidemiológica mundial en curso es el mejor ejemplo de ello. Ante la naturaleza del riesgo, los Estados nacionales intentan hacer frente a la situación epidémica que exige, para evitar aún más los bloqueos y alteraciones en la continuidad del flujo de capital que tendrían, modificar el estilo y los actos del poder. Como señala Badiou, el Estado debe imponer, no solamente a las masas populares sino también a la clase capitalista, restricciones importantes para salvar al capitalismo local: "guerra o epidemia, el Estado es obligado, incluso yendo más allá el juego normal de su naturaleza de clase, a aplicar prácticas tanto más autoritarias como más globales para evitar una catástrofe estratégica" (2020, p. 74). El paradigma inmunitario aquí se descifra en aquellas intervenciones que, aunque forzosas, intrusivas, autoritarias o, incluso, ilegales, se justifican como reparadoras: lo que es a la vez un veneno, puede ser una cura.

Pero antes de este escenario de crisis epidemiológica, ya nos encontramos con un abanico de tecnologías con las que el poder gestiona la "amenaza" y que también se caracterizan por una filosofía social que tiene como base la indemnización. En esta línea se encuentran, por ejemplo, en Argentina:

- Las políticas destinadas a morigerar los efectos de la exclusión y desafectación social: programas como la AUH (Asignación Universal por Hijo), Argentina Trabaja, entre otros, administran la inclusión a partir de una cuota mínima de acceso al consumo como instancia filiatoria.

- Los programas destinados a paliar los efectos del desempleo y que suponen la adquisición de destrezas y conocimientos para un mercado altamente competitivo, fluctuante y expulsivo (11).

- Los programas de lucha contra la delincuencia que, tomando los criterios de la epidemiología y la prevención, vuelven cada vez más densa la vigilancia del espacio público y cada vez más preciso el control sobre los movimientos de los individuos.

Una gestión diferencial de la desigualdad exige, por un lado, un orden espacial enteramente transparente, en el que se visibilizan completamente las jerarquías,

(11) Los programas de capacitación para el empleo desarrollados desde mediados de los años 90 e intensificados post crisis del 2001 dan cuenta de esta condición. Y no estamos hablando solamente de programas de ejecución nacional, sino en todos los niveles. Por ejemplo, en la provincia de Córdoba, el “Programa Primer Paso", el "Primer Paso Aprendiz", “Por mí", “Córdoba por ellas”, entre otros. 
pertenencias, localizaciones y diferencias de su población, que requiere de ciertas condiciones securitarias para su producción y reproducción. Ello nos remite a un creciente proceso de vigilancia del espacio (público-privado); combinado con los más variados mecanismos regulatorios, de control y disciplinamiento (que van desde la instalación de cientos de cámaras de seguridad, cercamiento de zonas rojas o calientes, hasta la aplicación constante de, por ejemplo, "códigos de convivencia ciudadana" sobre una población cada vez más indefensa y con menos derechos civiles y políticos). Con ello, la persecución del "otro" pasa a normalizarse y se vuelve parte del tejido cotidiano de la existencia.

Han señala, justamente, que la estrategia asiática para enfrentar al virus COVID-19 apostó fuertemente por la vigilancia digital(12), que colabora, simultáneamente, con el sinsentido de concebir el orden político desde la existencia de una -inviolable - esfera privada, y con la reducción del único sentido que puede adquirir el espacio público como espacio posible y pasible de ser vigilado. Precisa el autor:

Toda la infraestructura para la vigilancia digital ha resultado ser ahora sumamente eficaz para contener la epidemia. Cuando alguien sale de la estación de Pekín es captado automáticamente por una cámara que mide su temperatura corporal. Si la temperatura es preocupante todas las personas que iban sentadas en el mismo vagón reciben una notificación en sus teléfonos móviles. No en vano el sistema sabe quién iba sentado dónde en el tren (...). Entonces la vida puede llegar a ser muy peligrosa (Han, 2020, pp. 100-101).

El espacio, en general, y la ciudad, en particular, que se organiza con estos principios teje la otra plataforma oscura de la racionalidad neoliberal: el Estado Vigilante. Este asume casi exclusivamente el ejercicio de mantener a raya a los sectores potencialmente peligrosos, es decir, los pobres en general, pero a quienes se organizan y resisten en particular. Estado vigilante y Estado de (in)Seguridad controlan y equilibran un umbral, ese punto o ese grado en donde la precarización y la vigilancia pueden transformarse en rebelión. En palabras de Butler:

Esta forma particular de poder prepara el terreno para crear la necesidad de seguridad como ideal político máximo, un ideal que sirve para acumular poder dentro del Estado y de las instituciones empresaria-

(12) Señalando una discusión sobre las posibilidades de reestructuración de la economía global en torno a las últimas innovaciones digitales, Morozov (2019) dice que "Silicon Valley sigue siendo un poderoso laboratorio de nuevas, y quizá mejores, soluciones de mercado. Ningún otro sector tiene un papel tan destacado en el horizonte del imaginario capitalista occidental, ni ofrece un campo tan prometedor de mitologías regeneradoras" (2019, p. 35). 
les a la par que produce un nuevo tipo de sujeto. Las poblaciones se definen ahora, no por la crítica y la resistencia, sino por su necesidad de paliar su inseguridad y, por ende, de valorizar formas de policía y control estatal, promesas de inversión global e instituciones de gobernanza global (2016, p. 14)

\section{Reflexiones finales: inmunidad, política y derecho}

El escenario abierto a nivel global y local por la racionalidad neoliberal da lugar a un modo de gobernanza que se organiza a partir de riesgos universalizados y la cooperación por amenaza, lo cual se traduce en un claro debilitamiento del Estado de Derecho y los órdenes democráticos. Como bien dice Esposito: "desde el punto de vista legal, el decreto de urgencia, que se ha aplicado durante mucho tiempo, incluso en los casos en que no sería necesario, empuja la política hacia procedimientos excepcionales (...)" (2020, párr. 2). La suspensión de garantías y derechos para individuos y grupos sociales se expresa como un modo de gestión corriente en el mundo contemporáneo y agita los fantasmas del autoritarismo.

Perpetrándose en nombre de la libertad y la justicia, una condición política verdaderamente nociva se instala ahí donde el lenguaje de la seguridad, tanto física como económica, se presenta como un juego en el que lo que unos ganan es directamente proporcional a lo que otros pierden. En este escenario, el ejercicio de la violencia consiste en procesos degradantes dirigidos a invalidar la seguridad y los derechos que merecen ciertas vidas y estilos de vida.

En este escenario, urge reinventar el orden desde un paradigma político, jurídico y económico centrado en la solidaridad, la democracia, la diversidad y la igual valía de todas las vidas. La desigualdad y el miedo conducen - como muestra la historia - a la guerra y/o al totalitarismo. Frente a este peligro inminente, la política democrática - que se gesta en las luchas sociales que promueven un empoderamiento colectivo- es el único resguardo frente a los poderes totalizantes de las tecnocracias y sus elites.

\section{Bibliografía}

Acosta, A. (2016). Maldiciones, herejías y otros milagros de la economía extractivista. Tabula Rasa, 24 (pp. 25-55).

Avalle, G. (2017). Técnicas de gubernamentalidad y degradación de derechos. Análisis de programas laborales en Argentina. Sudamérica, 1(6) (pp. 114-136).

Azamar Alonso, A. y Carrillo González, G. (2017). Extractivismo y deuda ecológica en América Latina. Luna Azul, 45 (pp. 400-401). 
Azarian, F. (2016). Neoliberalismo y Biopolíticas. Resistencias carreras en Córdoba. Anuario XVI del Centro de Investigaciones Jurídicas y Sociales de la Facultad de Derecho de la Universidad Nacional de Córdoba. Córdoba: La Ley.

Azarian, F.; Villegas Guzmán, S. M. y Whitney, L. (2017). Judicializar, institucionalizar, formalizar: el estado frente a las luchas por la tierra en Córdoba (Argentina). XVIII Congreso Nacional y VIII Latinoamericano de Sociología Jurídica "Nuevos contextos en América Latina, derechos y sociedades en crisis. Tendencias y alternativas" (Mendoza, 11, 12 y 13 de octubre).

Badiou, A. (2020). Sobre la situación epidémica. En P. Amadeo (comp.), Sopa de Wuhan (pp. 67-78). La Plata: ASPO.

Brown, W. (2015). El pueblo sin atributos: la secreta revolución del neoliberalismo. Barcelona: Malpaso.

Butler, J. (2016). Prefacio. En I. Lorey, Estado de inseguridad. Gobernar la precariedad. Madrid: Traficantes de sueños-mapas.

Comisión Económica para América Latina y el Caribe (CEPAL) (2020). La Agenda 2030 para el Desarrollo Sostenible en el nuevo contexto mundial y regional. Santiago de Chile: CEPAL. Recuperado de https://www.cepal.org/es/ publicaciones/45336-la-agenda-2030-desarrollo-sostenible-nuevo-contextomundial-regional-escenarios [Fecha de consulta: 06/04/2020].

Comisión Económica para América Latina y el Caribe (CEPAL) (2016). La matriz de la desigualdad social en América Latina. Santiago de Chile: CEPAL. Recuperado de https://www.cepal.org/es/publicaciones/40668-la-matriz-la-desigualdad-social-america-latina [Fecha de consulta: 06/04/2020].

Ciuffolini, M. A. (2010) Resistencias. Luchas Sociales Urbanas en Córdoba post2001. Córdoba: EDUCC.

Ciuffolini, M. A. (2017a). La Dinámica del Neoliberalismo y sus desplazamientos. Para una crítica inmanente en orden a su superación. Studia Politicae, 40 (pp. 85-101).

Ciuffolini, M. A. (2017b). Quien no se mueve no siente sus cadenas. Estudios sobre luchas político-sociales en la Córdoba Contemporánea. Córdoba: EDUCC.

Composto, C. y Navarro, M. L. (2014). Claves de lectura para comprender el despojo y las luchas por los bienes comunes naturales en América Latina. En C. Composto y M. L. Navarro (comps.), Territorios en disputa. Despojo capitalista, 
luchas en defensa de los bienes comunes naturales y alternativas emancipatorias para América Latina (pp. 33-75). CDMX: Bajo tierra.

Dávilo, B. (2018). Gobernar con la libertad, gobernar con la competencia: sobre la racionalidad gubernamental liberal y neoliberal. En B. Dávilo (Ed.), La libertad como tecnología de gobierno (pp. 39-66). Paraná: EDUNER.

De la Vega, C. (2010). Política habitacional y ciudadanía. El Programa "Mi Casa, Mi vida" en la ciudad de Córdoba, Argentina. Revista Encrucijada Americana, 1 (pp. 70-97).

De la Vega, C. (2017). Institucionalidad ambiental y procesos de conflictividad social: una mirada política en los casos de las provincias de Córdoba y La Rioja en Argentina. Estado \& Comunes, 2(5) (pp. 63-92).

De la Vega, C. (2019). Un salto desde el vacío: la clase y el "problema" de la heterogeneidad de los sectores subalternos. Actuel Marx Intervenciones, 26 (pp. 17-37).

De la Vega, C. (2020). Quien aún esté vivo, que no diga 'nunca'. Resistencias contemporáneas y luchas 'sin clases'. Perfiles Latinoamericanos, 28 (56). En prensa.

De la Vega, C. y Ciuffolini, M. A. (2019). ¡Aquí está la rosa, baila aquí! Un ejercicio sobre las claves y dimensiones de un análisis clasista de conflictos sociales. En D. Pérez Roig et. al. (comp.), Naturaleza, territorio y conflicto en la trama capitalista contemporánea (pp. 69-91). Ranelagh: Extramuros.

De la Vega, C.; Caccia, A.; Dellea, G. y Latimori, V. (2020). Una práctica de frontera. Las prácticas de responsabilidad social universitaria en la disputa con la gestión neoliberal de la ciencia. En H. Ferreyra (comp.), Miradas y voces de la Investigación Educativa II. Innovación educativa con miradas a la justicia social. Aportes desde la Investigación Educativa. En prensa. Córdoba: Red de Posgrados en Educación A.C-EDUCC.

De Lagasnerie, G. (2015). La última lección de Michel Foucault. Buenos Aires: FCE.

Espósito, R. (2011). Bios. Biopolítica y Filosofía. Buenos Aires: Amorrortu.

Esposito, R. (2020). Cuidados a ultranza. Ficción de la razón. Recuperado de https://ficciondelarazon.org/2020/03/13/roberto-esposito-cuidados-a-ultranza/ [Fecha de consulta: 31/03/2020].

Foucault, Michel (2000). Defender la sociedad. Buenos Aires: Fondo de Cultura Económica. 
Foucault, M. (2006). Seguridad, Territorio, Población. Curso de Michel Foucault en el collège de France (1977-1978). Buenos Aires: Fondo de Cultura Económica.

Foucault, M. (2007). Nacimiento de la biopolítica. Buenos Aires: FCE.

Foucault, M (2008). Historia de la sexualidad 1. La voluntad de saber. Buenos Aires: Siglo XXI.

Fraser, N. (2017). ¿Una Nueva Forma de Capitalismo? New Left Review, 106 (pp. 61-70).

Gago, V. y Mezzadra, S. (2015). Para una crítica de las operaciones extractivas del capital. Patrón de acumulación y luchas sociales en el tiempo de la financiarización. Nueva Sociedad, 255 (pp. 38-52).

Gray, J. (1994). Liberalismo. Madrid: Aliaza.

Hardt, M. y Negri, A. (2020). Imperio, veinte años después. New Left Review, 120 (pp. 71-98).

Harvey, D. (2020). Política anticapitalista en tiempos de coronavirus. En P. Amadeo (comp.), Sopa de Wuhan (pp. 79-96). La Plata: ASPO.

Hayek, F. (2008). Los Fundamentos de la Libertad. Madrid: Unión editorial.

Laval, C. y Dardot, P. (2013). La Nueva Razón del Mundo. Barcelona: Gedisa.

Lazzarato, M. (2013). La fábrica del hombre endeudado. Buenos Aires: Amorrortu.

Lazzarato, M. (2015). Gobernar a través de la deuda. Buenos Aires: Amorrortu.

Lorey, I. (2016). Estado de inseguridad. Gobernar la precariedad. Madrid: Traficantes de sueños-mapas.

Saccucci, E. (2018). Estudio de las dimensiones de la precariedad en cinco conflictos de la ciudad de Córdoba. Espacio abierto, 26(4), (pp. 111-130).

Torres, M. (2019). Neoliberalismo y subjetividad. Bogotá: Universidad Pedagógica Nacional.

Fecha de recepción: 31-03-2020

Fecha de aceptación: 13-07-2020 
